F-47

\section{Screening strategies for Hepatitis C Virus} elimination in Italy

L.A. Kondili ${ }^{1}$, A. Marcellusi ${ }^{2}$, I. Gamkrelidze ${ }^{3}$, S. Blach $^{3}$, S. Robbins ${ }^{3}$, A. Craxì ${ }^{4}$, M. Puoti ${ }^{5}$, S. Vella $^{1}$, H. Razavi ${ }^{3}$, F.S. Mennini ${ }^{2}$

${ }^{1}$ Center for Global Health, Istituto Superiore di Sanità, Rome

${ }^{2}$ CEIS-Economic Evaluation and HTA (EEHTA),

Faculty of Economics, University of Rome "Tor

Vergata", Rome

${ }^{3}$ Center for Disease Analysis, CDA Foundation |

Polaris Observatory, Lafayette, Colorado

${ }^{4}$ Gastroenterolgy and Liver Unit, Biomedical

Department of Internal Medicine and Specialties

(Di.Bi.M.I.S.), University of Palermo, Palermo

${ }^{5}$ Department of Infectious Disease, Niguarda Cà

Granda Hospital, Milan

Introduction and aim: Cost-effective screening strategies are needed to make HCV elimination a reality. HCV is more prevalent in the older Italian population, so we aimed to determine if birth cohort-based screening is cost-effective in Italy.

Method: A Markov model was populated with Italian data to quantify the annual $\mathrm{HCV}$-infected population by liver disease stage, sex, and age. The economic impact was evaluated quantifying HCV infection medical costs (screening, antiviral treatment, liver-related complications) and health effects denominated as quality-adjusted-life years (QALYs). Prevalence of undiagnosed, $\mathrm{HCV}$ infection was used to calculate the number of HCV antibody screens needed annually. The cost-effectiveness threshold was set at $€ 25,000$. Modeled HCV infection outcomes (disease burden, medical costs, health effects) were assessed under the status quo and a scenario to achieve the WHO elimination targets. The screening strategies included universal or targeted screening by birth cohort: the 1948-78 cohort, the 1958-78 cohort, and graduated birth cohort screening (birth years 1958-78 over 2021-23, 1948-78 over $2024-27$, and $1948-84$ over $2028-30$ ).

Results: All screening scenarios were found to be highly costeffective ( $<€ 3,000 /$ QALY gained) vs. the status quo. The 1948-78 birth cohort screening scenario was the least costly, with $€ 5.5$ billion in total medical costs by 2031 . This was $€ 24.7$ million less than screening in the 1958-78 birth cohort, $€ 37.6$ million less than universal screening, and $€ 55.3$ million less than graduated screening. Screening the 1948-78 birth cohort would gain approximately 140,000 QALYs by 2031 , vs. $134,000,127,000$, and 123,000 QALYs for the universal, 1958-78 birth cohort, and graduated birth cohort, respectively.

Conclusion: Implementing screening in the 1948-78 birth cohort was the most cost-effective option with the greatest reductions in overall disease burden by 2030 . This strategy should be considered to sustain Italy's momentum towards achieving HCV elimination goals.

https://doi.org/10.1016/j.dld.2018.11.163
F-48

Changes in ${ }^{13} \mathrm{C}$-aminopyrine breath test predict liver-related events and death in patients with HCV-related previous decompensated child A5 or child A6 to B cirrhosis who achieve SVR after DAA therapy

S. Petta ${ }^{1}$, F. Rini ${ }^{2}$, V. Calvaruso ${ }^{1}$, C. Cammà ${ }^{1}$, S. Ciminnisi $^{1}$, V. Di Marco ${ }^{1}$, E. Giannini ${ }^{2}$, S. Grimaudo $^{1}$, R.M. Pipitone ${ }^{1}$, A. Craxì ${ }^{1}$

${ }^{1}$ Sezione di Gastroenterologia e Epatologia, PROMISE, University of Palermo, Italy

2 Dipartimento di Medicina Interna, Unità di

Gastroenterologia, Ospedale Policlinico San Martino, Università di Genova, Genova, Italy

Background and aims: The effects of SVR by DAA on decompensation and liver deaths in patients with advanced cirrhosis is less clearcut. We used ${ }^{13} \mathrm{C}$-aminopyrine breath test(ABT), a noninvasive method evaluating microsomal hepatocellular function, at baseline and after SVR, to assess whether its changes can predict liver-related outcomes after DAA treatment in patients with advanced HCV-cirrhosis.

Methods:50 consecutive patients with HCV cirrhosis were enrolled. Twenty-one patients (42\%) were in Child Pugh stage B79, 22 (44\%) C-P A6, and 5 (14\%) C-P A5. All C-P A5 patients had a recent history of decompensation. $\mathrm{ABT}$ was performed at baseline and 12 weeks after the end of antiviral therapy. Patients received sofosbuvir-based regimens.

Results: ABT was available for all 50 patients at baseline. The $120 \%$ cumulative dose was directly associated at regression analysis only with albumin levels $(p=0.001)$. ABT was available at follow-up week 12 for 41 patients (FUW12), all with SVR, and followed for a median of 25.2 months (range 12.2-32.1 months). Three patients did not start therapy, 3 could not be tested at FUW12 and 2 died during the observation period. At univariate analysis, changes from FUW12 to baseline 120\%cumulative dose ABT(HR 0.97, 95\%C.I. 0.95-0.99; $p=0.02$ ) -but not baseline and FUW12 120\%cumulative dose ABT-, baseline MELD (HR 1.16, 95\%C.I. 0.97-1.38; $p=0.09$ ), and hepatic encephalopathy at FUW12 (HR 8.70, 95\%C.I. 0.88-85.6; $p=0.06$ ) were the only variables associated with occurrence of liver-related events/death ( 7 cases), while delta 120\%cumulative dose ABT (HR 0.67, 95\%C.I. 0.47-0.95; $p=0.02$ ) and FUW12 hepatic encephalopathy (HR 17.2, 95\%C.I. $1.32-224.3 ; p=0.02$ ) remained the only independent predictors at multivariate Cox regression analysis. The AUC of delta $120 \%$ cumulative dose ABT for predicting liver-related events/death was good (0.87, 95\%C.I. $0.75-0.97$ ), with a delta $\geq 0 \%$ well discriminating patients at lower versus patients at higher risk of liver-related events-death $(p<0.001)$.

Conclusions: In patients with advanced HCV cirrhosis who achieve SVR with DAA, changes in $120 \%$ cumulative dose ABT assists in assessing the residual likelihood of liver-related events and deaths after viral cure.

https://doi.org/10.1016/j.dld.2018.11.164 